\title{
Impacto del aula invertida en el proceso de enseñanza-aprendizaje sobre los mapas de Karnaugh
}

\author{
Impact of Flipped Classroom in the Teaching-Learning Process on Karnaugh's Maps
}

Impacto da sala de aula invertida no processo de ensino-aprendizagem nos mapas de Karnaugh

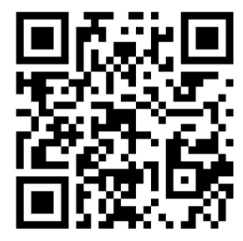

Ricardo Adán Salas-Rueda

Universidad Nacional Autónoma de México Instituto de Ciencias Aplicadas y Tecnología

Ciudad de México, México ricardoadansalasrueda@hotmail.com https://orcid.org/0000-0002-4188-4610

Recibido • Received • Recebido: 17 / 07 / 2019

Corregido • Revised • Revisado: 14 / 03 / 2021

Aceptado • Accepted • Aprovado: 07 / 04 / 2021

\begin{abstract}
Resumen: Esta investigación cuantitativa analiza el impacto del aula invertida en el proceso de enseñanza-aprendizaje sobre los mapas de Karnaugh, para ello considera la ciencia de datos, las redes neuronales y el aprendizaje automático (regresión lineal). En el aprendizaje automático, la sección de entrenamiento utilizó el $60 \%, 70 \%$ y $80 \%$ de la muestra para evaluar las hipótesis de investigación. Los mapas de Karnaugh permiten identificar la función lógica que minimiza el número de componentes electrónicos utilizados durante la construcción de los circuitos digitales. El objetivo general es implementar y analizar el uso sobre el aula invertida en el proceso educativo sobre los mapas de Karnaugh. La muestra está compuesta por 26 estudiantes de la Licenciatura Ejecutiva en Sistemas Computacionales que cursaron la asignatura Lógica secuencial y combinatoria en una universidad mexicana. Esta investigación propone la implementación del aula invertida por medio de la consulta de videos YouTube (antes de la clase), el uso del protoboard en forma colaborativa (durante la clase) y el empleo del software Crocodile Clips (después de la clase). El protoboard es una placa de pruebas que permite la construcción de circuitos digitales y el software Crocodile Clips permite realizar diversas simulaciones sobre el funcionamiento de los circuitos digitales. Los resultados del aprendizaje automático indican que el aula invertida influye positivamente el rol activo y la motivación del estudiantado durante el proceso de aprendizaje. La ciencia de datos identifica 6 modelos predictivos sobre el aula invertida por medio de la técnica árbol de decisión. La información sobre el perfil del estudiantado (sexo y edad) y el uso de las herramientas (videos YouTube, protoboard y software Crocodile Clips) permiten la construcción de estos modelos. Asimismo, las redes neuronales identifican los aspectos del aula invertida que influyen en el rol activo y la motivación del estudiantado. Por último, en este estudio se ha comprobado que el aula invertida mejora las condiciones de enseñanza-aprendizaje por medio de la consulta de videos YouTube, el uso del protoboard en forma colaborativa y el empleo del software Crocodile Clips.
\end{abstract}

Palabras claves: Aula invertida; tecnología educativa; ciencia de datos; enseñanza; aprendizaje superior. 
http://doi.org/10.15359/ree.25-2.14

http://www.una.ac.cr/educare

educare@una.ac.cr

\begin{abstract}
This quantitative design research analyzes flipped classroom's effects in the teachinglearning process on Karnaugh's maps considering data science, neural networks, and machine learning (linear regression). In machine learning, the training section used $60 \%, 70 \%$, and $80 \%$ of the sample to test the research hypotheses. Karnaugh's maps allow identifying the logical function that minimizes the number of electronic components used during the digital circuits' construction. The general aim is to implement and analyze the use of the flipped classroom in the educational process on Karnaugh's maps. The sample is composed of 26 students from the Executive Degree in Computational Systems. The students were enrolled in the Sequential and Combinatorial Logic course at a Mexican university. This research proposes the implementation of a flipped classroom using the consultation of YouTube videos (before the class), use of the protoboard in a collaborative mode (during the class), and use of the Crocodile Clips software (after the class). The protoboard is a test plate that allows the construction of digital circuits, and Crocodile Clips software allows performing various simulations on digital circuits' operation. Machine learning results indicate that flipped classrooms positively influence the student's active role and motivation during the learning process. Data science identifies six predictive models on flipped classrooms employing the decision tree technique. The information about the student's profile (sex and age) and use of the tools (YouTube videos, breadboard, and Crocodile Clips software) allow the construction of these models. Likewise, neural networks identify the flipped classroom aspects that influence the student's active role and motivation. Finally, flipped classrooms improve the teaching-learning conditions through the consultation of YouTube videos, the use of the protoboard in a collaborative way, and the Crocodile Clips software.
\end{abstract}

Keywords: Flipped classroom; educational technology; data science; teaching; higher learning.

Resumo: Esta pesquisa quantitativa analisa o impacto da sala de aula invertida no processo de ensino-aprendizagem em mapas de Karnaugh, considerando os dados da ciência, redes neuronais e aprendizagem automático (regressão linear). No aprendizado automático, a seção de treinamento usou $60 \%, 70 \%$ e $80 \%$ da amostra para avaliar as hipóteses de pesquisa. Os mapas de Karnaugh permitem identificar a função lógica que minimiza o número de componentes eletrónicos utilizados durante a construção de circuitos digitais. O objetivo geral é implementar e analisar o uso da sala de aula invertida no processo educacional sobre os mapas de Karnaugh. A amostra é constituída por 26 estudantes de Licenciatura Executiva em Sistemas de Computação que terminaram o curso de Lógica seqüencial e combinacional em uma universidade mexicana. Esta pesquisa propõe a implementação da sala de aula invertida através de consulta de vídeos de YouTube (antes da aula), o uso de protoboard de forma colaborativa (durante a aula) e o uso de software Crocodile Clips (depois da aula). A placa de ensaio (protoboard) permite a construção de circuitos digitais e o software Crocodile Clips permite várias simulações sobre o funcionamento de circuitos digitais. Os resultados da aprendizagem automática indicam que a sala de aula invertida influencia positivamente o papel ativo e a motivação estudantil durante o processo de aprendizagem. Os dados da ciência identificam 6 modelos preditivos na sala de aula invertida por meio da técnica da árvore de decisão. As informações sobre o perfil de estudante (sexo e idade) e o uso das ferramentas (vídeos do YouTube, protoboard e software Crocodile Clips) permitem a construção desses modelos. Da mesma forma, as redes neuronais identificam os aspectos da sala de aula invertida que influenciam o papel ativo e a motivação do estudante. Finalmente, a sala de aula invertida melhora as condições de ensino e aprendizagem através de consulta vídeos do YouTube, a placa de ensaio de forma colaborativa e uso de software Crocodile Clips.

Palavras-chave: Sala de aula invertida; tecnologia educacional; ciência de dados; ensino; aprendizado superior. 


\section{Introducción}

Los avances de las tecnologías de la información y comunicación (TIC) están provocando que las instituciones educativas actualicen los modelos pedagógicos y las estrategias didácticas, con la finalidad de mejorar la asimilación del conocimiento y el desarrollo de las habilidades en el estudiantado (Akyuz, 2018; Boz y Adnan, 2017; Salas-Rueda et al., 2019; Samaniego et al., 2015). De hecho, el crecimiento acelerado de las herramientas tecnológicas basadas en internet está provocando el surgimiento de nuevos modelos para la enseñanza y el aprendizaje (Nelson et al., 2019; Reyes et al., 2017; Salas-Rueda, 2019; Tibi, 2018). En particular, el aula invertida es un modelo pedagógico que propone la realización de diversas actividades dentro y fuera del salón de clases a través del uso de las TIC (Urbina et al., 2015).

Los contenidos audiovisuales, las aplicaciones online, las plataformas web y los programas informáticos permiten la construcción de nuevos escenarios educativos centrados en el estudiantado (Kim, 2017). Por ejemplo, los videos YouTube facilitan la difusión de la información en cualquier momento y lugar (Smith et al., 2017). Asimismo, el uso de las aplicaciones web dentro y fuera del salón de clases propicia el rol activo del estudiantado durante el proceso de aprendizaje (Sharp y Hamil, 2018).

En el aula invertida, la consulta de los contenidos audiovisuales en internet permite a su público usuario obtener flexibilidad de tiempo y espacio (Akçayir y Akçayir, 2018). Incluso, la incorporación de los videos en las actividades escolares facilita la personalización del aprendizaje (Schwartz et al., 2016).

Asimismo, el aula invertida fomenta el trabajo colaborativo en el salón de clases con la finalidad de desarrollar las competencias digitales en el estudiantado (Akçayir y Akçayir, 2018). Del mismo modo, el alumnado utiliza el tiempo de clase para discutir, analizar y reflexionar sobre los temas de las asignaturas (Karabulut-Ilgu et al., 2018; Shih y Tsai, 2017; Zainuddin, 2018).

Hoy en día, el aula invertida está transformando la organización y realización de las actividades escolares a través de la tecnología (Schwartz et al., 2018; Shih y Tsai, 2017). En particular, esta investigación propone la implementación del aula invertida en el proceso de enseñanza-aprendizaje sobre los mapas de Karnaugh, por medio de la consulta de videos YouTube (antes de la clase), el uso del protoboard en forma colaborativa (durante la clase) y el empleo del software Crocodile Clips (después de la clase).

En la asignatura Lógica secuencial y combinatoria, el tema de los mapas de Karnaugh tiene un papel fundamental para facilitar la compresión sobre el uso de las compuertas lógicas en el contexto productivo y la construcción de los circuitos digitales con el mínimo de compuertas lógicas. Algunos de los videos sobre los mapas de Karnaugh utilizados en el aula invertida se localizan en https://www.youtube.com/hashtag/\%C3\%A1lgebrabooleana. 
http://doi.org/10.15359/ree.25-2.14

http://www.una.ac.cr/educare

educare@una.ac.cr

Las preguntas de investigación son:

- ¿Cuál es el impacto del aula invertida en el rol activo y la motivación del estudiantado durante el proceso de enseñanza-aprendizaje sobre los mapas de Karnaugh?

- ¿Cuáles son los modelos predictivos sobre el uso del aula invertida en el proceso educativo de los mapas de Karnaugh considerando el perfil del estudiantado, la consulta de videos YouTube antes de la clase, el uso del protoboard en forma colaborativa durante la clase y el empleo del software Crocodile Clips después de la clase?

- ¿Cuáles son los aspectos del aula invertida que influyen positivamente en el rol activo y la motivación del estudiantado durante el proceso de enseñanza-aprendizaje sobre los mapas de Karnaugh considerando las redes neuronales?

\section{Aula invertida}

El aula invertida es un modelo pedagógico que permite satisfacer las demandas y necesidades de la sociedad en el siglo XXI (Guy y Marquis, 2016; Schwartz et al., 2018). En particular, el aula invertida promueve el uso de una gran variedad de recursos didácticos y multimedia (p.ej., videos, contenidos audiovisuales, presentaciones digitales, evaluaciones en línea y aplicaciones web) durante el proceso de enseñanza-aprendizaje (Tanner y Scott, 2015).

En el aula invertida, el alumnado realiza diversas actividades fuera y dentro de la sesión presencial (Schwartz et al., 2018; Zainuddin, 2018). El estudiantado puede consultar los videos y las lecturas digitales en sus casas (Akçayir y Akçayir, 2018). Asimismo, el docente organiza e implementa actividades interactivas, dinámicas y colaborativas durante el tiempo del salón de clases (Karabulut-Ilgu etal., 2018; Kim, 2017). Por ejemplo, la incorporación de la videoconferencia en el aula invertida mejora el desempeño académico del estudianado (Urbina et al., 2015).

El aula invertida permite la construcción de entornos virtuales educativos centrados en el estudiantado (Tanner y Scott, 2015). El alumnado consulta los contenidos audiovisuales y recursos multimedia de los cursos en cualquier momento y lugar durante el aula invertida, por consiguiente, este modelo pedagógico facilita el aprendizaje personalizado (Tanner y Scott, 2015). Incluso, el personal docente crea actividades activas dentro y fuera del salón de clases considerando la taxonomía de Bloom (Yang, 2017).

Una de las ventajas del aula invertida está asociada con la flexibilidad de tiempo y espacio durante el proceso de enseñanza-aprendizaje (Guy y Marquis, 2016). Incluso, este modelo pedagógico mejora el rendimiento académico y la motivación del estudiantado (Yang, 2017).

El aula invertida ha sido implementada en diversos cursos sobre la informática (Guy y Marquis, 2016; Tanner y Scott, 2015), el inglés (Yang, 2017) y las matemáticas (Hodgson et al., 2017) con el propósito de ofrecer otras alternativas para planear, organizar y realizar el proceso de enseñanza-aprendizaje.

4 
En el curso Sistemas de información, el estudiantado de la Universidad Tennessee State consultó diversas lecturas digitales y podcasts antes de asistir a la sesión presencial y realizaron foros de discusión, evaluaciones en línea y ejercicios de forma colaborativa en el salón de clases (Guy y Marquis, 2016). Del mismo modo, el aula invertida facilitó el proceso de enseñanzaaprendizaje sobre los diagramas UML por medio de las lecturas digitales, las evaluaciones en líneas y las discusiones grupales en el salón de clases (Tanner y Scott, 2015).

En los cursos de inglés, el aula invertida permite la construcción de espacios virtuales educativos que promueven la creatividad y el rol activo del estudiantado (Yang, 2017). De hecho, este modelo pedagógico mejoró también las condiciones de enseñanza-aprendizaje sobre las matemáticas recurriendo al uso de videos antes de las sesiones presenciales y el trabajo colaborativo durante la resolución de los ejercicios en el salón de clases (Hodgson et al., 2017).

Por último, el personal docente está implementando el aula invertida en el proceso educativo con el propósito de desarrollar las competencias digitales y matemáticas, incrementar la motivación durante el aprendizaje y propiciar el rol activo del estudiantado (Karabulut-Ilgu et al., 2018; Zainuddin, 2018).

\section{Metodología}

Esta investigación cuantitativa analiza el impacto del aula invertida en el proceso de enseñanza-aprendizaje sobre los mapas de Karnaugh considerando la ciencia de datos, las redes neuronales y el aprendizaje automático (regresión lineal). Es importante mencionar que este tema tiene gran relevancia en los cursos de las matemáticas y la electrónica, debido a que el estudiantado aprende a reducir el número de compuertas lógicas utilizadas durante la construcción de los circuitos digitales. .

Por consiguiente, este estudio propone la incorporación de los videos YouTube, el protoboard y el software Crocodile Clips en las actividades escolares sobre los mapas de Karnaugh antes, durante y después de las sesiones presenciales. El protoboard es una placa de pruebas que permite la construcción de circuitos digitales y el software Crocodile Clips permite realizar la simulación sobre el funcionamiento de los circuitos digitales.

\section{Participantes}

La muestra está compuesta por 26 estudiantes, 17 hombres (65,38\%) y 9 mujeres $(34,62 \%)$, de la Licenciatura Ejecutiva en Sistemas Computacionales que cursaron la asignatura Lógica secuencial y combinatoria en una universidad mexicana durante el ciclo escolar 2015. La edad promedio del estudiantado es 26,69 años. Las clases en la modalidad ejecutiva están dirigidas a personas que estudian y trabajan. El tipo de muestreo es no probabilístico. 


\section{Procedimiento}

El procedimiento de esta investigación inició con el uso del modelo ADDIE en la unidad mapas de Karnaugh (ver Tabla 1). Este modelo permite analizar el contexto educativo con la finalidad de organizar las actividades escolares de los cursos (Salas Rueda et al., 2020).

Tabla 1: Modelo ADDIE

\begin{tabular}{|c|c|c|c|}
\hline No. & Etapa & Actividad & Descripción \\
\hline \multirow{3}{*}{1} & \multirow{3}{*}{ Análisis } & $\begin{array}{l}\text { Evaluación de } \\
\text { necesidades }\end{array}$ & $\begin{array}{l}\text { El alumnado de la asignatura Lógica secuencial y combinatoria } \\
\text { tiene dificultades para asimilar y utilizar el conocimiento sobre } \\
\text { los mapas de Karnaugh }\end{array}$ \\
\hline & & $\begin{array}{l}\text { Identificación del } \\
\text { problema }\end{array}$ & $\begin{array}{l}\text { El alumnado de la Licenciatura Ejecutiva en Sistemas } \\
\text { Computacionales necesita relacionar y aplicar los contenidos } \\
\text { teóricos sobre los mapas de Karnaugh en el contexto empresarial }\end{array}$ \\
\hline & & $\begin{array}{l}\text { Características del } \\
\text { estudiantado }\end{array}$ & $\begin{array}{l}\text { El alumnado de la Licenciatura Ejecutiva en Sistemas Computa- } \\
\text { cionales cursó la asignatura Lógica secuencial y combinatoria du- } \\
\text { rante el segundo cuatrimestre. Las clases en la modalidad ejecuti- } \\
\text { va están dirigidas a personas que estudian y trabajan }\end{array}$ \\
\hline \multirow{4}{*}{2} & \multirow{4}{*}{ Diseño } & $\begin{array}{l}\text { Definición de los } \\
\text { objetivos }\end{array}$ & $\begin{array}{l}\text { Comprender y utilizar los conceptos teóricos sobre los mapas de } \\
\text { Karnaugh en el contexto empresarial }\end{array}$ \\
\hline & & Plan instruccional & $\begin{array}{l}\text { Esta investigación propone el uso del aula invertida por medio } \\
\text { de la consulta de videos YouTube (antes de la clase), el uso del } \\
\text { protoboard en forma colaborativa (durante la clase) y el empleo } \\
\text { del software Crocodile Clips (después de la clase) }\end{array}$ \\
\hline & & $\begin{array}{l}\text { Identificación de } \\
\text { recursos }\end{array}$ & $\begin{array}{l}\text { Incorporación de los videos YouTube, el protoboard y el software } \\
\text { Crocodile Clips en las actividades escolares }\end{array}$ \\
\hline & & $\begin{array}{l}\text { Desarrollo de los } \\
\text { temas }\end{array}$ & $\begin{array}{l}\text { Uso de los contenidos teóricos sobre los mapas de Karnaugh } \\
\text { en ejercicios vinculados con la realidad (manejo de sensores y } \\
\text { diseño del sumador) }\end{array}$ \\
\hline 3 & Desarrollo & $\begin{array}{l}\text { Creación del ambiente } \\
\text { de aprendizaje }\end{array}$ & $\begin{array}{l}\text { El docente de la asignatura Lógica secuencial y combinatoria } \\
\text { organizó } 3 \text { prácticas de laboratorio sobre los mapas de Karnaugh } \\
\text { considerando el aula invertida }\end{array}$ \\
\hline 4 & Implementación & Asignatura & $\begin{array}{l}\text { El alumnado de la Licenciatura Ejecutiva en Sistemas } \\
\text { Computacionales cursó la asignatura Lógica secuencial y } \\
\text { combinatoria en una universidad mexicana durante el ciclo } \\
\text { escolar } 2015\end{array}$ \\
\hline 5 & Evaluación & $\begin{array}{l}\text { Análisis de la } \\
\text { información }\end{array}$ & $\begin{array}{l}\text { La herramienta Rapiminder permite analizar el uso del aula } \\
\text { invertida en el proceso de enseñanza-aprendizaje sobre los } \\
\text { mapas de Karnaugh por medio del aprendizaje automático } \\
\text { (regresión lineal), la ciencia de datos y las redes neuronales }\end{array}$ \\
\hline
\end{tabular}

Nota: Elaboración propia. 
http://doi.org/10.15359/ree.25-2.14

http://www.una.ac.cr/educare educare@una.ac.cr

La Tabla 2 describe las 3 prácticas de laboratorio realizadas en la asignatura Lógica secuencial y combinatoria.

Tabla 2: Prácticas de laboratorio considerando el uso del aula invertida

\begin{tabular}{|c|c|c|c|c|}
\hline No. & Tema & Antes de la clase & Durante la clase & Después de la clase \\
\hline 1 & $\begin{array}{l}\text { Mapas de Karnaugh para } \\
2 \text { variables }\end{array}$ & $\begin{array}{l}\text { Consulta los videos } \\
\text { YouTube sobre la reducción } \\
\text { de la función lógica para } 2 \\
\text { variables por medio de los } \\
\text { mapas de Karnaugh }\end{array}$ & $\begin{array}{l}\text { Con tu compañero o compañera de } \\
\text { clase construye un circuito digital en el } \\
\text { protoboard que active una alarma cuando } \\
\text { por lo menos } 1 \text { sensor esté activo. Utiliza } \\
\text { los mapas de Karnaugh para minimizar la } \\
\text { función lógica. }\end{array}$ & $\begin{array}{l}\text { Utiliza el software Crocodile } \\
\text { Clips para simular la función } \\
\text { lógica reducida (mapas de } \\
\text { Karnaugh) sobre el sumador } \\
\text { de } 2 \text { variables }\end{array}$ \\
\hline 2 & $\begin{array}{l}\text { Mapas de Karnaugh para } \\
3 \text { variables }\end{array}$ & $\begin{array}{l}\text { Consulta los videos YouTube } \\
\text { sobre la reducción de } \\
\text { la función lógica para } 3 \\
\text { variables por medio de los } \\
\text { mapas de Karnaugh }\end{array}$ & $\begin{array}{l}\text { Con tu compañero o compañera de } \\
\text { clase construye un circuito digital en el } \\
\text { protoboard que active una alarma cuando } \\
\text { por lo menos } 2 \text { sensores estén activos. } \\
\text { Utiliza los mapas de Karnaugh para } \\
\text { minimizar la función lógica. }\end{array}$ & $\begin{array}{l}\text { Utiliza el software Crocodile } \\
\text { Clips para simular la función } \\
\text { lógica reducida (mapas de } \\
\text { Karnaugh) sobre el sumador } \\
\text { de } 3 \text { variables }\end{array}$ \\
\hline 3 & $\begin{array}{l}\text { Mapas de Karnaugh para } \\
4 \text { variables }\end{array}$ & $\begin{array}{l}\text { Consulta los videos } \\
\text { YouTube sobre la reducción } \\
\text { de la función lógica para } 4 \\
\text { variables por medio de los } \\
\text { mapas de Karnaugh }\end{array}$ & $\begin{array}{l}\text { Con tu compañero o compañera de } \\
\text { clase construye un circuito digital en el } \\
\text { protoboard que active una alarma cuando } \\
\text { por lo menos } 3 \text { sensores estén activos. } \\
\text { Utiliza los mapas de Karnaugh para } \\
\text { minimizar la función lógica. }\end{array}$ & $\begin{array}{l}\text { Utiliza el software Crocodile } \\
\text { Clips para simular la función } \\
\text { lógica reducida (mapas de } \\
\text { Karnaugh) sobre el sumador } \\
\text { de } 4 \text { variables }\end{array}$ \\
\hline
\end{tabular}

Nota: Elaboración propia.

Esta investigación cuantitativa analizó el impacto de la consulta de videos YouTube antes de la clase, el uso del protoboard en forma colaborativa durante la clase y el empleo del software Crocodile Clips después de la clase (aula invertida) en el proceso de enseñanza-aprendizaje sobre los mapas de Karnaugh considerando los aspectos del rol activo y la motivación del estudiantado.

Las hipótesis de investigación sobre el uso del aula invertida y el rol activo del estudiantado son:

- Hipótesis 1 (H1): La consulta de videos YouTube antes de la clase influye positivamente el rol activo del estudiantado durante el proceso de aprendizaje sobre los mapas de Karnaugh.

- Hipótesis $2(\mathrm{H} 2)$ : El uso del protoboard en forma colaborativa durante la clase influye positivamente el rol activo del estudiantado durante el proceso de aprendizaje sobre los mapas de Karnaugh.

- Hipótesis 3 (H3): El empleo del software Crocodile Clips después de la clase influye positivamente el rol activo del estudiantado durante el proceso de aprendizaje sobre los mapas de Karnaugh. 
http://doi.org/10.15359/ree.25-2.14

http://www.una.ac.cr/educare

educare@una.ac.cr

Las hipótesis de investigación sobre el uso del aula invertida y la motivación del estudiantado son:

- Hipótesis 4 (H4): La consulta de videos YouTube antes de la clase influye positivamente la motivación del estudiantado durante el proceso de aprendizaje sobre los mapas de Karnaugh.

- Hipótesis 5 (H5): El uso del protoboard en forma colaborativa durante la clase influye positivamente la motivación del estudiantado durante el proceso de aprendizaje sobre los mapas de Karnaugh.

- Hipótesis 6 (H6): El empleo del software Crocodile Clips después de la clase influye positivamente la motivación del estudiantado durante el proceso de aprendizaje sobre los mapas de Karnaugh.

\section{Análisis de datos}

La herramienta Rapidminer permite realizar el cálculo del aprendizaje automático con el propósito de identificar las regresiones lineales que presentan el menor error al cuadrado. La sección de entrenamiento utilizó el 60\%,70\% y $80 \%$ de la muestra para calcular las regresiones lineales y evaluar las hipótesis sobre el uso del aula invertida. Por otro lado, la sección de evaluación utilizó el $40 \%, 30 \%$ y $20 \%$ de la muestra para identificar el error al cuadrado. De acuerdo con Salas-Rueda et al. (2021), este software está disponible en la siguiente dirección web: https://rapidminer.com/

Asimismo, la herramienta Rapidminer permite construir los modelos predictivos sobre el uso del aula invertida en el proceso de enseñanza-aprendizaje por medio de la ciencia de datos (técnica árbol de decisión). La información sobre el perfil del estudiantado (sexo y edad) y el uso de las herramientas (videos YouTube, protoboard y software Crocodile Clips) permiten la construcción de estos modelos.

Por último, la herramienta Rapidminer permite identificar los aspectos del aula invertida que influyen durante el proceso de enseñanza-aprendizaje sobre los mapas de Karnaugh por medio de las redes neuronales. La sección de entrenamiento con $60 \%$ de la muestra permite conocer la relación entre las variables del aula invertida y la sección de evaluación con 40\% de la muestra permite conocer la exactitud.

\section{Recolección de datos}

La recolección de datos se realizó por medio de la aplicación de un cuestionario al finalizar la unidad sobre los mapas de Karnaugh en la asignatura Lógica secuencial y combinatoria durante el ciclo escolar 2015. La Tabla 3 muestra este instrumento de medición. Los valores del factor de carga son: Consulta de videos YouTube (0.595), uso del protoboard en forma colaborativa (0.652), empleo del software Crocodile Clips (0.734), rol activo del estudiantado (0.384) y motivación del estudiantado (0.462). Asimismo, el composite reliability es 0,706.

8 
http://doi.org/10.15359/ree.25-2.14

http://www.una.ac.cr/educare educare@una.ac.cr

Tabla 3: Instrumento de medición

\begin{tabular}{|c|c|c|c|c|c|c|}
\hline No. & Variable & Dimensión & Pregunta & Respuesta & $\mathrm{n}$ & $\%$ \\
\hline \multirow{11}{*}{1} & \multirow{11}{*}{ Estudiante } & \multirow{3}{*}{ Sexo } & 1. ¿Cuál es tu sexo? & & & \\
\hline & & & & Hombre & 17 & $65.38 \%$ \\
\hline & & & & Mujer & 9 & $34.62 \%$ \\
\hline & & \multirow{8}{*}{ Edad } & 2. ¿Cuál es tu edad? & & & \\
\hline & & & & 22 años & 2 & $7.69 \%$ \\
\hline & & & & 23 años & 3 & $11.54 \%$ \\
\hline & & & & 24 años & 3 & $11.54 \%$ \\
\hline & & & & 25 años & 5 & $19.23 \%$ \\
\hline & & & & 26 años & 3 & $11.54 \%$ \\
\hline & & & & 27 años & 2 & $7.69 \%$ \\
\hline & & & & $\geq 28$ años & 8 & $30.77 \%$ \\
\hline \multirow{12}{*}{2} & \multirow{12}{*}{$\begin{array}{l}\text { Asimilación del } \\
\text { conocimiento }\end{array}$} & $\begin{array}{l}\text { Actividad antes de la } \\
\text { clase }\end{array}$ & \multicolumn{4}{|c|}{$\begin{array}{l}\text { 3. La consulta de videos YouTube antes de la clase facilita la asimilación del conocimiento } \\
\text { sobre los Mapas de Karnaugh }\end{array}$} \\
\hline & & & & Bastante (1) & 14 & $53.85 \%$ \\
\hline & & & & Mucho (2) & 9 & $34.62 \%$ \\
\hline & & & & Poco (3) & 3 & $11.54 \%$ \\
\hline & & $\begin{array}{l}\text { Actividad durante la } \\
\text { clase }\end{array}$ & \multicolumn{4}{|c|}{$\begin{array}{l}\text { 4. El uso del protoboard en forma colaborativa durante la clase facilita la asimilación del } \\
\text { conocimiento sobre los mapas de Karnaugh }\end{array}$} \\
\hline & & & & Bastante (1) & 17 & $65.38 \%$ \\
\hline & & & & Mucho (2) & 6 & $23.08 \%$ \\
\hline & & & & Poco (3) & 3 & $11.54 \%$ \\
\hline & & $\begin{array}{l}\text { Actividad después de } \\
\text { la clase }\end{array}$ & \multicolumn{4}{|c|}{$\begin{array}{l}\text { 5. El empleo del software Crocodile Clips después de la clase facilita la asimilación del } \\
\text { conocimiento sobre los mapas de Karnaugh }\end{array}$} \\
\hline & & & & Bastante (1) & 17 & $65.38 \%$ \\
\hline & & & & Mucho (2) & 8 & $30.77 \%$ \\
\hline & & & & Poco (3) & 1 & $3.85 \%$ \\
\hline \multirow{8}{*}{3} & \multirow{8}{*}{ Aula invertida } & Proceso de aprendizaje & \multicolumn{4}{|c|}{ 6. El aula invertida influye el rol activo del estudiantado durante el proceso de aprendizaje } \\
\hline & & & & Bastante (1) & 16 & $61.54 \%$ \\
\hline & & & & Mucho (2) & 10 & $38.46 \%$ \\
\hline & & & & Poco (3) & 0 & $0.00 \%$ \\
\hline & & & \multicolumn{4}{|c|}{ 7. El aula invertida influye la motivación del estudiantado durante el proceso de aprendizaje } \\
\hline & & & & Bastante (1) & 14 & $53.85 \%$ \\
\hline & & & & Mucho (2) & 12 & $46.15 \%$ \\
\hline & & & & Poco (3) & 0 & $0.00 \%$ \\
\hline
\end{tabular}

Nota: Elaboración propia. 
http://doi.org/10.15359/ree.25-2.14

http://www.una.ac.cr/educare

educare@una.ac.cr

\section{Resultados}

La Tabla 3 indica que la consulta de videos YouTube antes de la clase facilita bastante ( $\mathrm{n}=$ 14: $53,85 \%)$, mucho $(n=9: 34,62 \%)$ y poco $(n=3: 11,54 \%)$ la asimilación del conocimiento sobre los mapas de Karnaugh. Por consiguiente, el estudiantado de la Licenciatura Ejecutiva en Sistemas Computacionales tiene una percepción favorable sobre las actividades escolares realizadas antes de las sesiones presenciales. Asimismo, el uso del protoboard en forma colaborativa durante la clase facilita bastante $(n=17: 65,38 \%)$, mucho $(n=6: 23,08 \%)$ y poco $(n=3: 11,54 \%)$ la asimilación del conocimiento sobre los mapas de Karnaugh. Del mismo modo, la percepción del estudiantado sobre el uso del protoboard en el salón de clase es favorable. Por último, el empleo del software Crocodile Clips después de la clase facilita bastante $(n=17: 65,38 \%)$, mucho $(n=8: 30,77 \%)$ y poco ( $n=1: 3,85 \%$ ) la asimilación del conocimiento sobre los mapas de Karnaugh. Por consiguiente, la incorporación de este software en la asignatura Lógica secuencial y combinatoria es favorable.

A continuación, se presentan los resultados sobre el impacto del aula invertida en el proceso de enseñanza-aprendizaje sobre los mapas de Karnaugh considerando la ciencia de datos, las redes neuronales y el aprendizaje automático.

\section{Rol activo del estudiantado}

El aula invertida influye bastante $(n=16: 61,54 \%)$ y mucho $(n=10: 38,46 \%)$ en el rol activo del estudiantado durante el proceso de aprendizaje (ver Tabla 3). Del mismo modo, los resultados del aprendizaje automático (regresión lineal) con $60 \%, 70 \%$ y $80 \%$ de entrenamiento indican que las actividades antes, durante y después de la clase influyen positivamente el rol activo del estudiantado durante el proceso de aprendizaje sobre los mapas de Karnaugh (ver Tabla 4).

Tabla 4: Resultados sobre el rol activo del estudiantado

\begin{tabular}{lccccc}
\hline \multicolumn{1}{c}{ Hipótesis } & Entrenamiento & Regresión lineal & Conclusión & Valor $\mathrm{t}$ & Error al cuadrado \\
\hline H1: Consulta de videos & $60 \%$ & $\mathrm{y}=0.252 \mathrm{x}+0.949$ & Acepta: 0.252 & 1.420 & 0.308 \\
YouTube $\rightarrow$ rol activo del & $70 \%$ & $\mathrm{y}=0.166 \mathrm{x}+1.111$ & Acepta: 0.166 & 0.936 & 0.254 \\
estudiantado & $80 \%$ & $\mathrm{y}=0.156 \mathrm{x}+1.135$ & Acepta: 0.156 & 0.946 & 0.268 \\
\hline H2: Uso del protoboard en & $60 \%$ & $\mathrm{y}=0.062 \mathrm{x}+1.276$ & Acepta: 0.062 & 0.385 & 0.245 \\
forma colaborativa $\rightarrow$ rol & $70 \%$ & $\mathrm{y}=0.047 \mathrm{x}+1.317$ & Acepta: 0.047 & 0.299 & 0.236 \\
activo del estudiantado & $80 \%$ & $\mathrm{y}=0.016 \mathrm{x}+1.355$ & Acepta: 0.016 & 0.111 & 0.239 \\
\hline H3: Empleo del software & $60 \%$ & $\mathrm{y}=0.166 \mathrm{x}+1.125$ & Acepta: 0.166 & 0.806 & 0.243 \\
Crocodile Clips $\rightarrow$ rol activo & $70 \%$ & $\mathrm{y}=0.137 \mathrm{x}+1.189$ & Acepta: 0.137 & 0.687 & 0.229 \\
del estudiantado & $80 \%$ & $\mathrm{y}=0.079 \mathrm{x}+1.266$ & Acepta: 0.079 & 0.420 & 0.223 \\
\hline
\end{tabular}

Nota: Elaboración propia. 
http://doi.org/10.15359/ree.25-2.14

Los resultados del aprendizaje automático con $60 \%(0,252$, valor $\mathrm{t}=1,420), 70 \%(0,166$, valor $\mathrm{t}$ $=0,936)$ y $80 \%(0,156$, valor $t=0,946)$ de entrenamiento indican que la hipótesis 1 es aceptada (ver Tabla 4). Por consiguiente, la consulta de videos YouTube antes de la clase influye positivamente el rol activo del estudiantado durante el proceso de aprendizaje sobre los mapas de Karnaugh.

La Figura 1 muestra el modelo predictivo 1 sobre el aula invertida. En particular, este modelo permite identificar cómo influye el sexo, la edad y la realización de las actividades escolares antes de las sesiones presenciales en el rol activo del estudiantado. Por ejemplo, si el estudiantado considera que la consulta de videos YouTube antes de la clase facilita bastante la asimilación del conocimiento sobre los mapas de Karnaugh y tiene una edad > 30,5 años, entonces, el aula invertida influye bastante el rol activo del estudiantado durante el proceso de aprendizaje.

Figura 1: Modelo predictivo 1 sobre el aula invertida

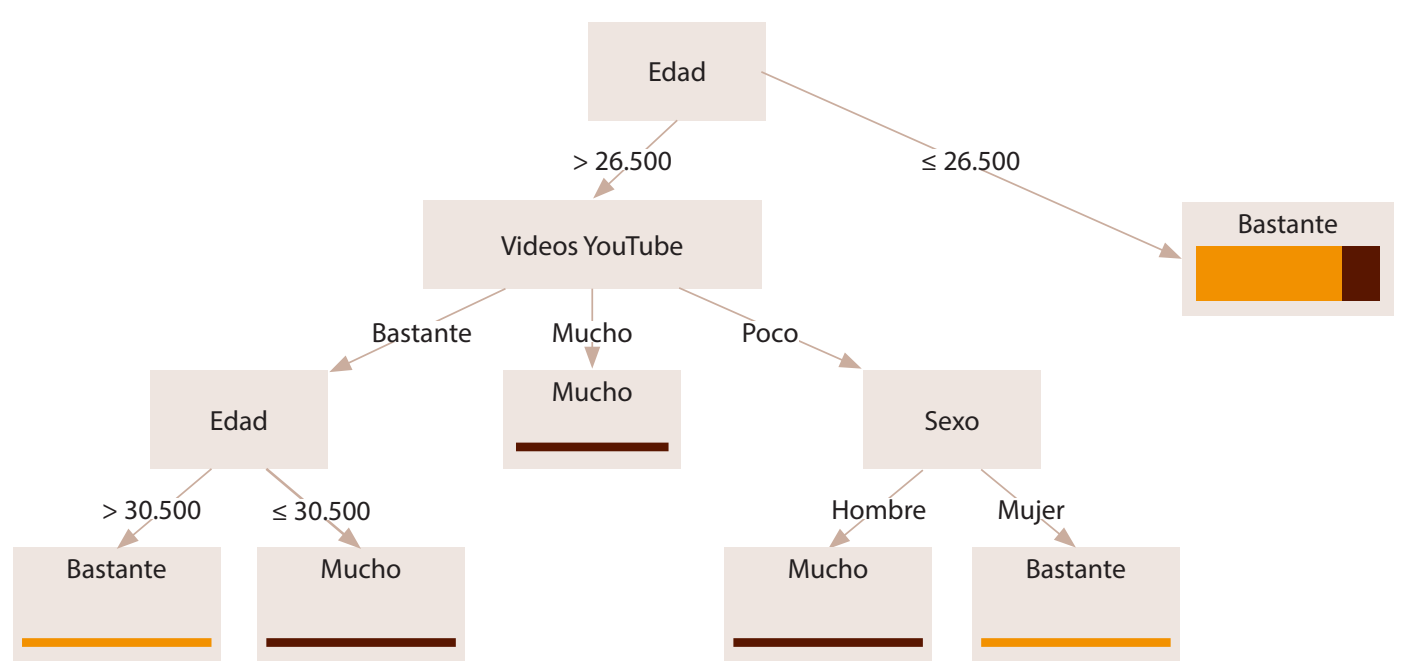

Nota: Elaboración propia por medio de la herramienta Rapidminer.

El modelo predictivo 1 presenta 5 condiciones sobre el uso del aula invertida con la exactitud del $88,46 \%$. Por ejemplo, si el estudiantado considera que la consulta de videos YouTube antes de la clase facilita mucho la asimilación del conocimiento sobre los mapas de Karnaugh y tiene una edad $\geq 26,5$ años, entonces, el aula invertida influye mucho el rol activo del estudiantado durante el proceso de aprendizaje (ver Figura 1).

Los resultados del aprendizaje automático con $60 \%(0,062$, valor $t=0,385), 70 \%(0,047$, valor $t=0,299)$ y $80 \%(0,016$, valor $t=0,111)$ de entrenamiento indican que la hipótesis 2 es aceptada (ver Tabla 4). Por consiguiente, el uso del protoboard en forma colaborativa durante la clase influye positivamente el rol activo del estudiantado durante el proceso de aprendizaje sobre los mapas de Karnaugh. 
http://doi.org/10.15359/ree.25-2.14

http://www.una.ac.cr/educare

educare@una.ac.cr

La Figura 2 muestra el modelo predictivo 2 sobre el aula invertida. Por ejemplo, si el estudiantado considera que el uso del protoboard en forma colaborativa durante la clase facilita bastante la asimilación del conocimiento sobre los mapas de Karnaugh, tiene una edad $\leq 26,5$ años y es hombre entonces el aula invertida influye bastante el rol activo del estudiantado durante el proceso de aprendizaje.

Figura 2: Modelo predictivo 2 sobre el aula invertida

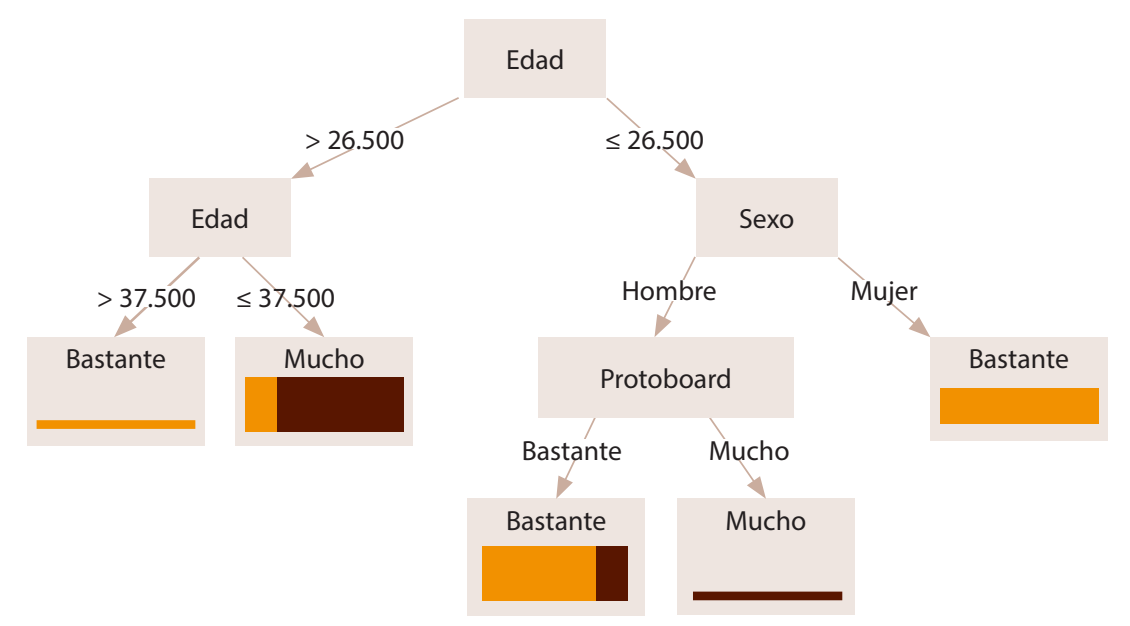

Nota: Elaboración propia por medio de la herramienta Rapidminer.

El modelo predictivo 2 presenta 2 condiciones sobre el uso del aula invertida con la exactitud del $84,62 \%$. En particular, este modelo permite identificar cómo influye el sexo, la edad y la realización de las actividades escolares durante de las sesiones presenciales en el rol activo del estudiantado. Por ejemplo, si el estudiantado considera que el uso del protoboard en forma colaborativa durante la clase facilita mucho la asimilación del conocimiento sobre los mapas de Karnaugh, tiene una edad $\leq 26,5$ años y es hombre, entonces, el aula invertida influye mucho el rol activo del estudiantado durante el proceso de aprendizaje (ver Figura 2).

Los resultados del aprendizaje automático con $60 \%(0,166$, valor $t=0,806), 70 \%(0,137$, valor $\mathrm{t}=0,687)$ y $80 \%(0,079$, valor $\mathrm{t}=0,420)$ de entrenamiento indican que la hipótesis 3 es aceptada (ver Tabla 4). Por lo tanto, el empleo del software Crocodile Clips después de la clase influye positivamente el rol activo del estudiantado durante el proceso de aprendizaje sobre los mapas de Karnaugh.

La Figura 3 muestra el modelo predictivo 3 sobre el uso del aula invertida. Por ejemplo, si el estudiantado considera que el empleo del software Crocodile Clips después de la clase facilita bastante la asimilación del conocimiento sobre los mapas de Karnaugh y tiene una edad $>$ 27,5 años, entonces, el aula invertida influye bastante el rol activo del estudiantado durante el proceso de aprendizaje. 
Figura 3: Modelo predictivo 3 sobre el aula invertida

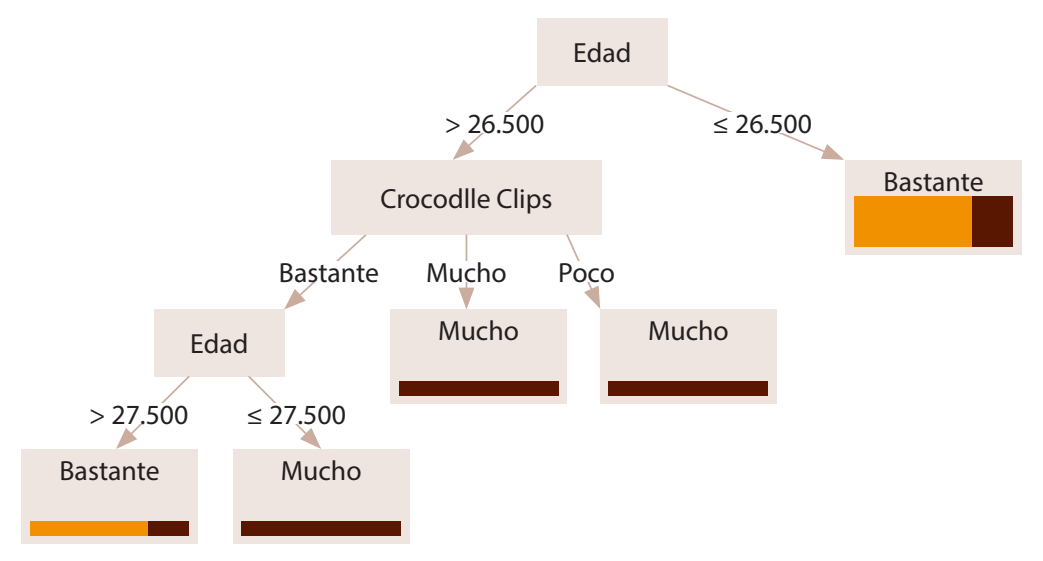

Nota: Elaboración propia por medio de la herramienta Rapidminer.

El modelo predictivo 3 presenta 4 condiciones sobre el uso del aula invertida con la exactitud del 84,62\%. En particular, este modelo permite identificar cómo influye el sexo, la edad y la realización de las actividades escolares después de las sesiones presenciales en el rol activo del estudiantado. Por ejemplo, si el estudiantado considera que el empleo del software Crocodile Clips después de la clase facilita mucho la asimilación del conocimiento sobre los mapas de Karnaugh y tiene una edad > 26,5 años entonces el aula invertida influye mucho el rol activo del estudiantado durante el proceso de aprendizaje (ver Figura 3).

La red neuronal utilizó la sección de entrenamiento con $60 \%$ de la muestra ( $n=16$ estudiantes) con la finalidad de identificar el impacto de las actividades escolares en el proceso de enseñanza-aprendizaje sobre los mapas de Karnaugh. En particular, los videos YouTube antes de la clase $(0,017)$, el uso del protoboard en forma colaborativa durante la clase $(1,000)$ y el empleo del software Crocodile Clips después de la clase $(0,560)$ influyen positivamente el rol activo del estudiantado durante el proceso de aprendizaje sobre los mapas de Karnaugh.

\section{Motivación del estudiantado}

El aula invertida influye bastante $(n=14: 53,85 \%)$ y mucho $(n=12: 46,15 \%)$ la motivación del estudiantado durante el proceso de aprendizaje (ver Tabla 3). Asimismo, los resultados del aprendizaje automático con $60 \%, 70 \%$ y $80 \%$ de entrenamiento indican que las actividades antes, durante y después de la clase influyen positivamente la motivación del estudiantado durante el proceso de aprendizaje sobre los mapas de Karnaugh (ver Tabla 5). 
http://doi.org/10.15359/ree.25-2.14

http://www.una.ac.cr/educare

educare@una.ac.cr

Tabla 5: Resultados sobre la motivación del estudiantado

\begin{tabular}{lccccc}
\hline \multicolumn{1}{c}{ Hipótesis } & Entrenamiento & Regresión lineal & Conclusión & Valor t & Error al cuadrado \\
\hline H4: Consulta de videos & $60 \%$ & $y=0.168 x+0.966$ & Acepta: 0.168 & 1.026 & 0.504 \\
$\begin{array}{l}\text { YouTube } \rightarrow \text { motivación del } \\
\text { estudiantado }\end{array}$ & $70 \%$ & $y=0.124 x+1.125$ & Acepta: 0.124 & 0.718 & 0.377 \\
& $80 \%$ & $y=0.046 x+1.307$ & Acepta: 0.046 & 0.278 & 0.325 \\
\hline H5: Uso del protoboard & $60 \%$ & $y=0.276 x+0.817$ & Acepta: 0.276 & 2.181 & 0.531 \\
en forma colaborativa & $70 \%$ & $y=0.190 x+1.047$ & Acepta: 0.190 & 1.297 & 0.354 \\
$\begin{array}{l}\rightarrow \text { motivación del } \\
\text { estudiantado }\end{array}$ & $80 \%$ & $y=0.194 x+1.093$ & Acepta: 0.194 & 1.338 & 0.326 \\
\hline $\begin{array}{l}\text { H6: Empleo del } \\
\text { software Crocodile }\end{array}$ & $60 \%$ & $y=0.166 x+1.000$ & Acepta: 0.166 & 0.907 & 0.511 \\
$\begin{array}{l}\text { Clips } \rightarrow \text { motivación del } \\
\text { estudiantado }\end{array}$ & $70 \%$ & $y=0.051 x+1.258$ & Acepta: 0.051 & 0.263 & 0.364 \\
\hline
\end{tabular}

Nota: Elaboración propia.

Los resultados del aprendizaje automático con $60 \%(0,168$, valor $t=1,026), 70 \%(0,124$, valor $t=0,718)$ y $80 \%(0,046$, valor $t=0,278)$ de entrenamiento indican que la hipótesis 4 es aceptada (ver Tabla 5). Por lo tanto, la consulta de videos YouTube antes de la clase influye positivamente la motivación del estudiantado durante el proceso de aprendizaje sobre los mapas de Karnaugh.

La Figura 4 muestra el modelo predictivo 4 sobre el aula invertida. Por ejemplo, si el estudiantado considera que la consulta de videos YouTube antes de la clase facilita bastante la asimilación del conocimiento sobre los mapas de Karnaugh y tiene una edad $\leq 30$ años entonces el aula invertida influye bastante la motivación del estudiantado durante el proceso de aprendizaje.

Figura 4: Modelo predictivo 4 sobre el aula invertida

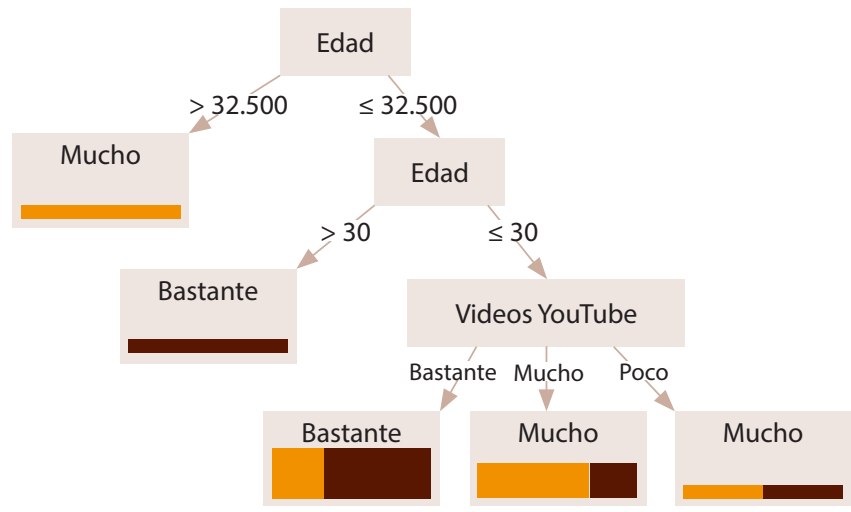

Nota: Elaboración propia por medio de la herramienta Rapidminer. 
http://doi.org/10.15359/ree.25-2.14

El modelo predictivo 4 presenta 3 condiciones sobre el uso del aula invertida con la exactitud del 69,23\%. En particular, este modelo permite identificar cómo influye el sexo, la edad y la realización de las actividades escolares antes de las sesiones presenciales en la motivación del estudiantado. Por ejemplo, si el estudiantado considera que la consulta de videos YouTube antes de la clase facilita mucho la asimilación del conocimiento sobre los mapas de Karnaugh y tiene una edad $\leq 30$ años, entonces, el aula invertida influye mucho la motivación del estudiantado durante el proceso de aprendizaje (ver Figura 4).

Los resultados del aprendizaje automático con $60 \%(0,276$, valor $t=2,181), 70 \%(0,190$, valor $\mathrm{t}=1,297)$ y $80 \%(0,194$, valor $\mathrm{t}=1.338)$ de entrenamiento indican que la hipótesis 5 es aceptada (ver Tabla 5). Por consiguiente, el uso del protoboard en forma colaborativa durante la clase influye positivamente la motivación del estudiantado durante el proceso de aprendizaje sobre los mapas de Karnaugh.

La Figura 5 muestra el modelo predictivo 5 sobre el aula invertida. Por ejemplo, el uso del protoboard en forma colaborativa durante la clase facilita bastante la asimilación del conocimiento sobre los mapas de Karnaugh y tiene una edad $\leq 30$ años, entonces, el aula invertida influye bastante la motivación del estudiantado durante el proceso de aprendizaje.

Figura 5: Modelo predictivo 5 sobre el aula invertida

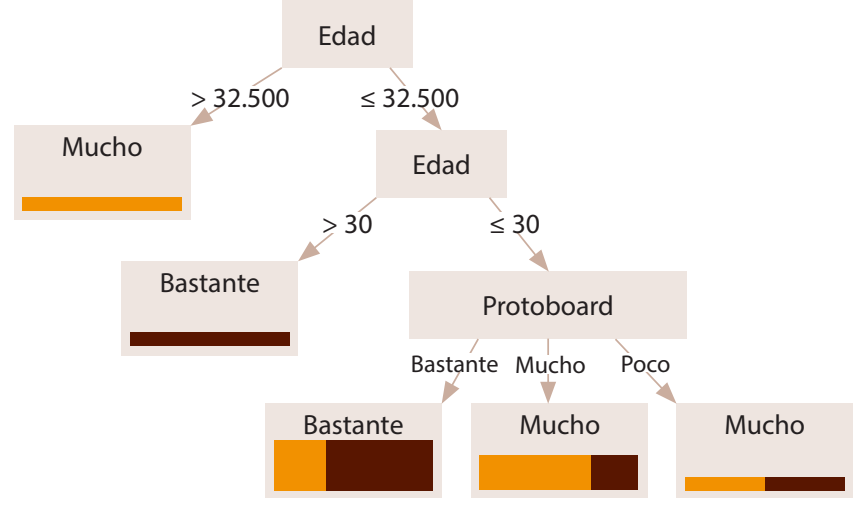

Nota: Elaboración propia por medio de la herramienta Rapidminer.

El modelo predictivo 5 presenta 3 condiciones sobre el uso del aula invertida con la exactitud del 69,23\%. En particular, este modelo permite identificar cómo influye el sexo, la edad y la realización de las actividades escolares durante las sesiones presenciales en la motivación del estudiantado. Por ejemplo, el uso del protoboard en forma colaborativa durante la clase facilita mucho la asimilación del conocimiento sobre los mapas de Karnaugh y tiene una edad $\leq 30$ años entonces el aula invertida influye mucho la motivación del estudiantado durante el proceso de aprendizaje (ver Figura 5). 
http://doi.org/10.15359/ree.25-2.14

http://www.una.ac.cr/educare

educare@una.ac.cr

Los resultados del aprendizaje automático con $60 \%(0,166$, valor $t=0,907), 70 \%(0,051$, valor $\mathrm{t}=0,263)$ y $80 \%(0,079$, valor $\mathrm{t}=0,420)$ de entrenamiento indican que la hipótesis 6 es aceptada (ver Tabla 5). Por lo tanto, el empleo del software Crocodile Clips después de la clase influye positivamente la motivación del estudiantado durante el proceso de aprendizaje sobre los mapas de Karnaugh.

La Figura 6 muestra el modelo predictivo 6 sobre el aula invertida. Por ejemplo, el empleo del software Crocodile Clips después de la clase facilita bastante la asimilación del conocimiento sobre los mapas de Karnaugh y tiene una edad $\leq 30$ años entonces el aula invertida influye bastante la motivación del estudiantado durante el proceso de aprendizaje.

Figura 6: Modelo predictivo 6 sobre el aula invertida

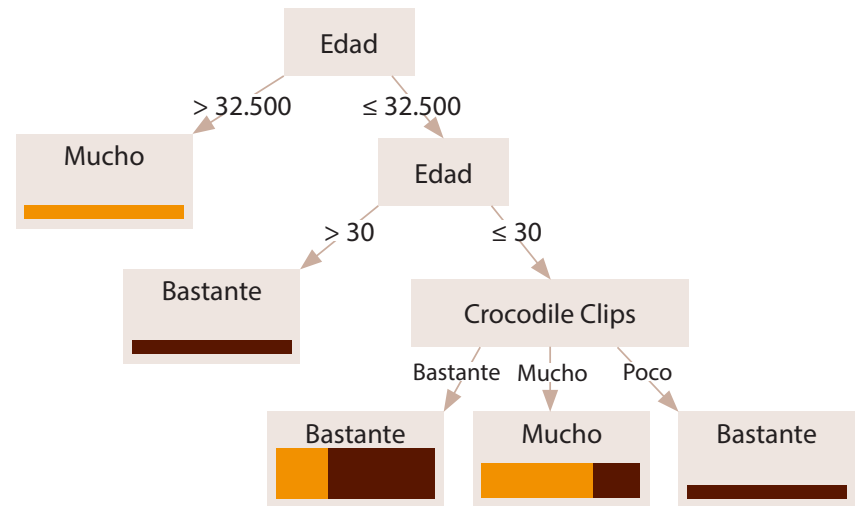

Nota: Elaboración propia por medio de la herramienta Rapidminer.

El modelo predictivo 6 presenta 3 condiciones sobre el uso del aula invertida con la exactitud del 69,23\%. En particular, este modelo permite identificar cómo influye el sexo, la edad y la realización de las actividades escolares después de las sesiones presenciales en la motivación del estudiantado. Por ejemplo, el empleo del software Crocodile Clips después de la clase facilita mucho la asimilación del conocimiento sobre los mapas de Karnaugh y tiene una edad $\leq 30$ años entonces el aula invertida influye mucho la motivación del estudiantado durante el proceso de aprendizaje (ver Figura 6).

La red neuronal utilizó la sección de entrenamiento con $60 \%$ de la muestra ( $\mathrm{n}=16$ estudiantes) con la finalidad de identificar el impacto de las actividades escolares en el proceso de enseñanza-aprendizaje sobre los mapas de Karnaugh. En particular, los videos YouTube antes de la clase $(0,936)$, el uso del protoboard en forma colaborativa durante la clase $(0,061)$ y el empleo del software Crocodile Clips después de la clase $(0,692)$ influyen positivamente la motivación del estudiantado durante el proceso de aprendizaje sobre los mapas de Karnaugh. 


\section{Discusión}

El aula invertida permite mejorar las condiciones de enseñanza-aprendizaje por medio de la incorporación de la tecnología en las actividades escolares (Shih y Tsai, 2017; Zainuddin, 2018). En particular, el alumnado de la asignatura Lógica secuencial y combinatoria considera que la consulta de videos YouTube antes de la clase $(n=14: 53,85 \%)$, el uso del protoboard en forma colaborativa durante la clase $(n=17: 65,38 \%)$ y el empleo del software Crocodile Clips después de la clase $(n=17: 65,38 \%)$ facilita bastante la asimilación del conocimiento sobre los mapas de Karnaugh.

Esta investigación cuantitativa comparte las ideas de diversos estudios (p.ej., KarabulutIlgu et al., 2018; Schwartz et al., 2018) relacionados con la importancia del aula invertida para lograr la innovación en el contexto educativo. En particular, las actividades antes, durante y después de la clase favorecen un rol activo y la motivación del estudiantado.

Similar a Guy y Marquis (2016), la revisión de los contenidos audiovisuales en la casa mejoró el proceso de enseñanza-aprendizaje en la modalidad del aula invertida. Los resultados del aprendizaje automático con $60 \%(0,252), 70 \%(0,166)$ y $80 \%(0,156)$ de entrenamiento indican que la consulta de videos YouTube antes de la clase influye positivamente en el rol activo del estudiantado durante el proceso de aprendizaje sobre los mapas de Karnaugh. Asimismo, la ciencia de datos identifica 5 condiciones del modelo predictivo 1 sobre la consulta de videos YouTube antes de la clase con la exactitud del 88,46\% por medio de la técnica árbol de decisión.

Los contenidos audiovisuales tienen un papel fundamental en el aula invertida debido a que estos recursos multimedia facilitan la difusión de la información (Guy y Marquis, 2016; Schwartz et al., 2016). Los resultados del aprendizaje automático con $60 \%(0,168), 70 \%(0,124)$ y $80 \%(0,046)$ de entrenamiento indican que la consulta de videos YouTube antes de la clase influye positivamente la motivación del estudiantado durante el proceso de aprendizaje sobre los mapas de Karnaugh. Además, la técnica árbol de decisión identifica 3 condiciones del modelo predictivo 4 sobre la consulta de videos YouTube antes de la clase con la exactitud del 69,23\%.

En el aula invertida, las actividades escolares realizadas durante la clase permiten la asimilación del conocimiento y fomentan el trabajo en equipo (Shih y Tsai, 2017; Tanner y Scott, 2015). Como lo mencionan Tanner y Scott (2015), la realización de las actividades colaborativas en el salón de clases favorece el proceso de aprendizaje. Los resultados del aprendizaje automático con $60 \%(0,062), 70 \%(0,047)$ y $80 \%(0,016)$ de entrenamiento indican que el uso del protoboard en forma colaborativa durante la clase influye positivamente el rol activo del estudiantado durante el proceso de aprendizaje sobre los mapas de Karnaugh. Asimismo, la ciencia de datos identifica 2 condiciones del modelo predictivo 2 sobre el uso del protoboard en forma colaborativa durante la clase con la exactitud del 84,62\% por medio de la técnica árbol de decisión. 
http://doi.org/10.15359/ree.25-2.14

http://www.una.ac.cr/educare

educare@una.ac.cr

Del mismo modo, los resultados del aprendizaje automático con $60 \%(0,276), 70 \%(0,190)$ y $80 \%(0,194)$ de entrenamiento indican que el uso del protoboard en forma colaborativa durante la clase influye positivamente la motivación del estudiantado durante el proceso de aprendizaje sobre los mapas de Karnaugh. Además, la técnica árbol de decisión identifica 3 condiciones del modelo predictivo 5 sobre el uso del protoboard en forma colaborativa durante la clase con la exactitud del $69,23 \%$.

El aula invertida promueve el aprendizaje personalizado a través de la realización de diversas actividades escolares fuera del salón de clases (Akçayir y Akçayir, 2018). Como lo menciona Salas Rueda (2020), el aula invertida permite la organización y realización de las actividades escolares después de la sesión presencial con la finalidad de mejorar las condiciones de enseñanza-aprendizaje. Los resultados del aprendizaje automático con $60 \%(0,166), 70 \%$ $(0,137)$ y $80 \%(0,079)$ de entrenamiento indican que el empleo del software Crocodile Clips después de la clase influye positivamente un rol activo del estudiantado durante el proceso de aprendizaje sobre los mapas de Karnaugh. Asimismo, la ciencia de datos identifica 4 condiciones del modelo predictivo 3 sobre el empleo del software Crocodile Clips después de la clase con una exactitud del 84,62\% por medio de la técnica árbol de decisión.

Las aplicaciones tecnológicas permiten innovar las actividades escolares en el siglo XXI (Karabulut-llgu et al., 2018; Salas-Rueda et al., 2016; Sharp y Hamil, 2018). Los resultados del aprendizaje automático con $60 \%(0,166), 70 \%(0,051)$ y $80 \%(0,079)$ de entrenamiento indican que el empleo del software Crocodile Clips después de la clase influye positivamente la motivación del estudiantado durante el proceso de aprendizaje sobre los mapas de Karnaugh. Además, la técnica árbol de decisión identifica 3 condiciones del modelo predictivo 6 sobre el empleo del software Crocodile Clips después de la clase con la exactitud del 69,23\%.

Las redes neuronales indican que el uso del protoboard en forma colaborativa durante la clase influye positivamente el rol activo del estudiantado. Del mismo modo, la consulta los videos YouTube antes de la clase influyen positivamente la motivación del estudiantado.

El aula invertida permite mejorar el proceso de enseñanza-aprendizaje por medio de la tecnología (Kim, 2017; Shih y Tsai, 2017; Urbina et al., 2015). En particular, la consulta de videos YouTube antes de la clase, el uso del protoboard en forma colaborativa durante la clase y el empleo del software Crocodile Clips después de la clase facilitan el proceso de enseñanzaaprendizaje sobre los mapas de Karnaugh.

\section{Conclusión}

El aula invertida es un modelo pedagógico que se apoya en la tecnología para crear nuevos espacios educativos. En particular, la consulta de videos YouTube antes de la clase, el uso del 
http://doi.org/10.15359/ree.25-2.14

protoboard en forma colaborativa durante la clase y el empleo del software Crocodile Clips después de la clase se ha comprobado con este estudio que mejoran el proceso de enseñanzaaprendizaje sobre los mapas de Karnaugh.

Los resultados del aprendizaje automático con 60\%, 70\% y $80 \%$ indican que las actividades realizadas fuera del salón de clases (consulta de videos YouTube y empleo del software Crocodile Clips) y el trabajo colaborativo realizado en las clases presenciales por medio del protoboard influyen positivamente el rol activo y la motivación del estudiantado.

La técnica árbol de decisión identifica 6 modelos predictivos sobre el uso del aula invertida en el proceso de enseñanza-aprendizaje sobre los mapas de Karnaugh con una exactitud superior al 69,20\%, lo cual permite conocer cómo influye la realización de las actividades escolares en la asignatura Lógica secuencial y combinatoria. Asimismo, las redes neuronales indican que el uso del protoboard en forma colaborativa durante la clase influye positivamente en el rol activo del estudiantado y la consulta de los videos YouTube antes de la clase influye positivamente la motivación del estudiantado.

Los resultados de esta investigación recomiendan la incorporación de las herramientas digitales y los recursos multimedia en el proceso de enseñanza-aprendizaje considerando el modelo pedagógico aula invertida. En particular, los videos YouTube antes de la clase y el software Crocodile Clips después de la clase permiten la construcción de nuevos escenarios educativos. Asimismo, el uso del protoboard en las sesiones presenciales fomenta el trabajo colaborativo durante la construcción de los circuitos digitales.

Las limitaciones de esta investigación se encuentran circunscritas en el tamaño de la muestra y en el uso de los videos YouTube, el protoboard y el software Crocodile Clips en el contexto educativo. Por consiguiente, las futuras investigaciones pueden analizar el uso de las lecturas digitales y podcast antes de la sesión presencial, la incorporación de las aplicaciones web, los juegos digitales en el salón de clases, y el empleo de las redes sociales y los exámenes en línea después de la sesión presencial.

Las implicaciones de este estudio son la búsqueda, la selección y el uso de las TIC para actualizar las prácticas educativas dentro y fuera del salón de clases. Asimismo, el aula invertida permite la planeación, organización e implementación de actividades escolares centradas en el estudiantado. Por último, el aula invertida mejora las condiciones de enseñanza-aprendizaje por medio de la incorporación de las TIC en las actividades escolares.

\section{Referencias}

Akçayir, G. y Akçayir, M. (2018). The flipped classroom: A review of its advantages and challenges. Computers \& Education, 126, 334-345. https://doi.org/10.1016/j.compedu.2018.07.021 
http://doi.org/10.15359/ree.25-2.14

http://www.una.ac.cr/educare

educare@una.ac.cr

Akyuz, D. (2018). Measuring technological pedagogical content knowledge (TPACK) through performance assessment. Computers \& Education, 125, 212-225. https://doi.org/10.1016/j. compedu.2018.06.012

Boz, B. y Adnan, M. (2017). How do freshman engineering students reflect an online calculus course? International Journal of Education in Mathematics, Science and Technology (IJEMST), 5(4), 262-278. https://www.ijemst.net/index.php/ijemst/article/view/99/100

Guy, R. y Marquis, G. (2016). The flipped classroom: A comparison of student performance using instructional videos and podcasts versus the lecture-based model of instruction. Issues in Informing Science and Information Technology, 13, 1-13. https://doi.org/10.28945/3461

Hodgson, T. R., Cunningham, A., McGee, D., Kinne, L. J. y Murphy, T. J. (2017). Assessing behavioral engagement in flipped and non-flipped mathematics classrooms: Teacher abilities and other potential factors. International Journal of Education in Mathematics, Science and Technology (IJEMST), 5(4), 248-261. https://www.ijemst.net/index.php/ijemst/issue/ view/22

Karabulut-Ilgu, A., Jaramillo Cherrez, N.y Hassall, L. (2018). Flipping to engage students: Instructor perspectives on flipping large enrollment courses. Australasian Journal of Educational Technology, 34(4), 123-137. https://doi.org/10.14742/ajet.4036

Kim, D. (2017). Flipped interpreting classroom: Flipping approaches, student perceptions and design considerations. The Interpreter and Translator Trainer, 11(1), 38-55. https://doi.org/1 $\underline{0.1080 / 1750399 X .2016 .1198180}$

Nelson, M. J., Voithofer, R. y Cheng, S.-L. (2019). Mediating factors that influence the technology integration practices of teacher educators. Computers \& Education, 128, 330-344. https:// doi.org/10.1016/j.compedu.2018.09.023

Reyes, V. C., Jr., Reading, C., Doyle, H. y Gregory, S. (2017). Integrating ICT into teacher education programs from a TPACK perspective: Exploring perceptions of university lecturers. Computers \& Education, 115, 1-19. https://doi.org/10.1016/j.compedu.2017.07.009

Salas-Rueda, R.-A. (2019). Construction and evaluation of a web application for the educational process on normal distribution considering the science of data and machine learning. Research in Learning Technology, 27, 1-24. https://doi.org/10.25304/rlt.v27.2085

Salas Rueda, R. A. (2020). Use of the flipped classroom to design creative and active activities in the field of computer science. Creativity studies, 13(1), 136-151. https://doi.org/10.3846/ cs.2020.10336 
http://doi.org/10.15359/ree.25-2.14

Salas-Rueda, R.-A., Gamboa-Rodríguez, F., Salas-Rueda, É.-P. y Salas-Rueda, R. D. (2020). Diseño de una aplicación web para el proceso educativo sobre el uso del logaritmo en el campo de las matemáticas financieras. Texto Livre: Linguajem e Tecnologia, 13(1), 65-81. https:// doi.org/10.17851/1983-3652.13.1.65-81

Salas-Rueda, R.-A., Ramírez-Ortega, J.y Eslava-Cervantes, A.-L. (2021). Use of the collaborative wall to improve the teaching-learning conditions in the bachelor of visual arts. Contemporary Educational Technology, 13(1), 1-10. https://doi.org/10.30935/cedtech/8711

Salas-Rueda, R.-A., Salas-Rueda, E.-P. y Salas-Rueda, R.-D. (2019). Diseño y uso de una aplicación web para el campo de la estadística considerando el modelo Assure y la ciencia de datos. Texto Livre: Linguagem e Tecnologia, 12(1), 48-71. https://doi.org/10.17851/19833652.12.1.48-71

Salas Rueda, R. A., Vázquez Estupiñán, J. de J. y Lugo García, J. L. (2016). Uso del avatar en el proceso de enseñanza-aprendizaje sobre las aplicaciones de las derivadas. Revista de Comunicación de la SEECI, 20(39), 72-88. https://doi.org/10.15198/seeci.2016.39.72-88

Samaniego, G., Marqués, L. y Gisbert, M. (2015). El profesorado universitario y el uso de entornos virtuales de aprendizaje. Campus Virtuales, 4(2), 50-58. http://uajournals.com/ojs/index. php/campusvirtuales/article/view/84/92

Schwartz, T. A., Ajazi, E. y Monaco, J. (2018). Findings from a survey of statistics and biostatistics instructors in the health sciences who teach using an online or flipped format. Journal of Statistics Education, 26(2), 143-148. https://doi.org/10.1080/10691898.2018.1484675

Schwartz, T. A., Andridge, R. R., Sainani, K. L., Stangle, D. K.y Neely, M. L. (2016). Diverse perspectives on a flipped biostatistics classroom. Journal of Statistics Education, 24(2), 74-84. https://doi. org/10.1080/10691898.2016.1192362

Sharp, L. A. y Hamil, M. (2018). Impact of a web-based adaptive supplemental digital resource on student mathematics performance. Online Learning, 22(1),81-92.https://doi.org/10.24059/ olj.v22i1.1133

Shih, W.-L y Tsai, C.-Y. (2017). Students' perception of a flipped classroom approach to facilitating online project-based learning in marketing research courses. Australasian Journal of Educational Technology, 33(5), 32-49. https://doi.org/10.14742/ajet.2884

Smith, C., Crocker, S. y Allman, T. (2017). Reading between the lines: Accessing information via YouTube's automatic captioning. Online Learning, 21(1), 115-131. https://doi.org/10.24059/ olj.v21i1.823 
http://doi.org/10.15359/ree.25-2.14

http://www.una.ac.cr/educare

educare@una.ac.cr

Tanner, M. y Scott, E. (2015). A flipped classroom approach to teaching systems analysis, design and implementation. Journal of Information Technology Education: Research, 14, 219-241. https://doi.org/10.28945/2266

Tibi, M. H. (2018). Computer science students' attitudes towards the use of structured and unstructured discussion forums in online courses. Online Learning, 22(1), 93-106. https:// doi.org/10.24059/olj.v22i1.995

Urbina, S., Arrabal, M., Conde, M., Ordinas, C. y Rodríguez, S. (2015). Flipped classroom a través de videoconferencia: Un proyecto de innovación docente. Campus Virtuales, 4(2), 60-65. https://dialnet.unirioja.es/servlet/articulo?codigo $=5237332$

Yang, C. C. R. (2017). An investigation of the use of the'flipped classroom' pedagogy in secondary english language classrooms. Journal of Information Technology Education: Innovations in Practice, 16, 1-20. https://doi.org/10.28945/3635

Zainuddin, Z. (2018). Students' learning performance and perceived motivation in gamified flipped-class instruction. Computers \& Education, 126, 75-88. https://doi.org/10.1016/j. compedu.2018.07.003 